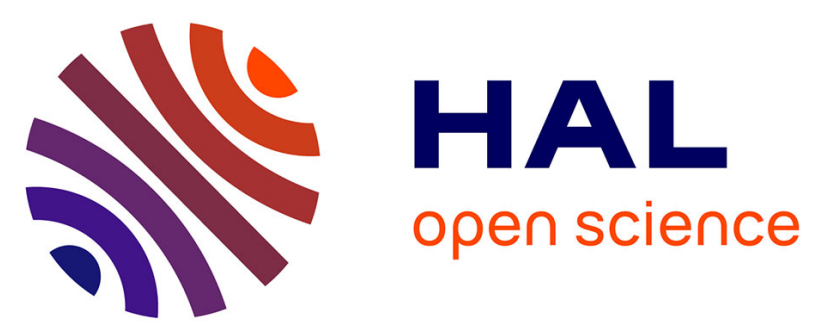

\title{
Comparison of human parechovirus and enterovirus detection frequencies in cerebrospinal fluid samples collected over a 5-year period in Edinburgh - HPeV type 3 identified as the most common picornavirus type
} Heli Harvala, Jaśmina Kondracka, Chloe Leanne Mcintyre, E Carol Mcwilliam Leitch, Kate E. Templeton, Peter Simmonds

\section{To cite this version:}

Heli Harvala, Jaśmina Kondracka, Chloe Leanne Mcintyre, E Carol Mcwilliam Leitch, Kate E. Templeton, et al.. Comparison of human parechovirus and enterovirus detection frequencies in cerebrospinal fluid samples collected over a 5-year period in Edinburgh - HPeV type 3 identified as the most common picornavirus type. Journal of Medical Virology, 2011, 83 (5), pp.889. 10.1002/jmv.22023 . hal-00624171

\section{HAL Id: hal-00624171 \\ https://hal.science/hal-00624171}

Submitted on 16 Sep 2011

HAL is a multi-disciplinary open access archive for the deposit and dissemination of scientific research documents, whether they are published or not. The documents may come from teaching and research institutions in France or abroad, or from public or private research centers.
L'archive ouverte pluridisciplinaire HAL, est destinée au dépôt et à la diffusion de documents scientifiques de niveau recherche, publiés ou non, émanant des établissements d'enseignement et de recherche français ou étrangers, des laboratoires publics ou privés. 
Comparison of human parechovirus and enterovirus detection frequencies in cerebrospinal fluid samples collected over a 5-year period in Edinburgh - HPeV type 3 identified as the most common picornavirus type

\begin{tabular}{|r|l|}
\hline Journal: & Journal of Medical Virology \\
\hline Manuscript ID: & JMV-10-2109.R1 \\
\hline Diley - Manuscript type: & Research Article \\
\hline Author: & 02-Nov-2010 \\
\hline & $\begin{array}{l}\text { Complete List of Authors: } \\
\text { McWintre, Chloe } \\
\text { Templeton, Katch, E Royal Infirmary Edinburgh, Department of } \\
\text { Medical Microbiology, Center of Infectious Diseases } \\
\text { Simmonds, Peter; Edinburgh, CID }\end{array}$ \\
\hline Keywords: & Sepsis, meningitis, surveillance, serotype, neonatal \\
\hline
\end{tabular}

\section{SCHOLARONE ${ }^{\text {M }}$ Manuscripts}




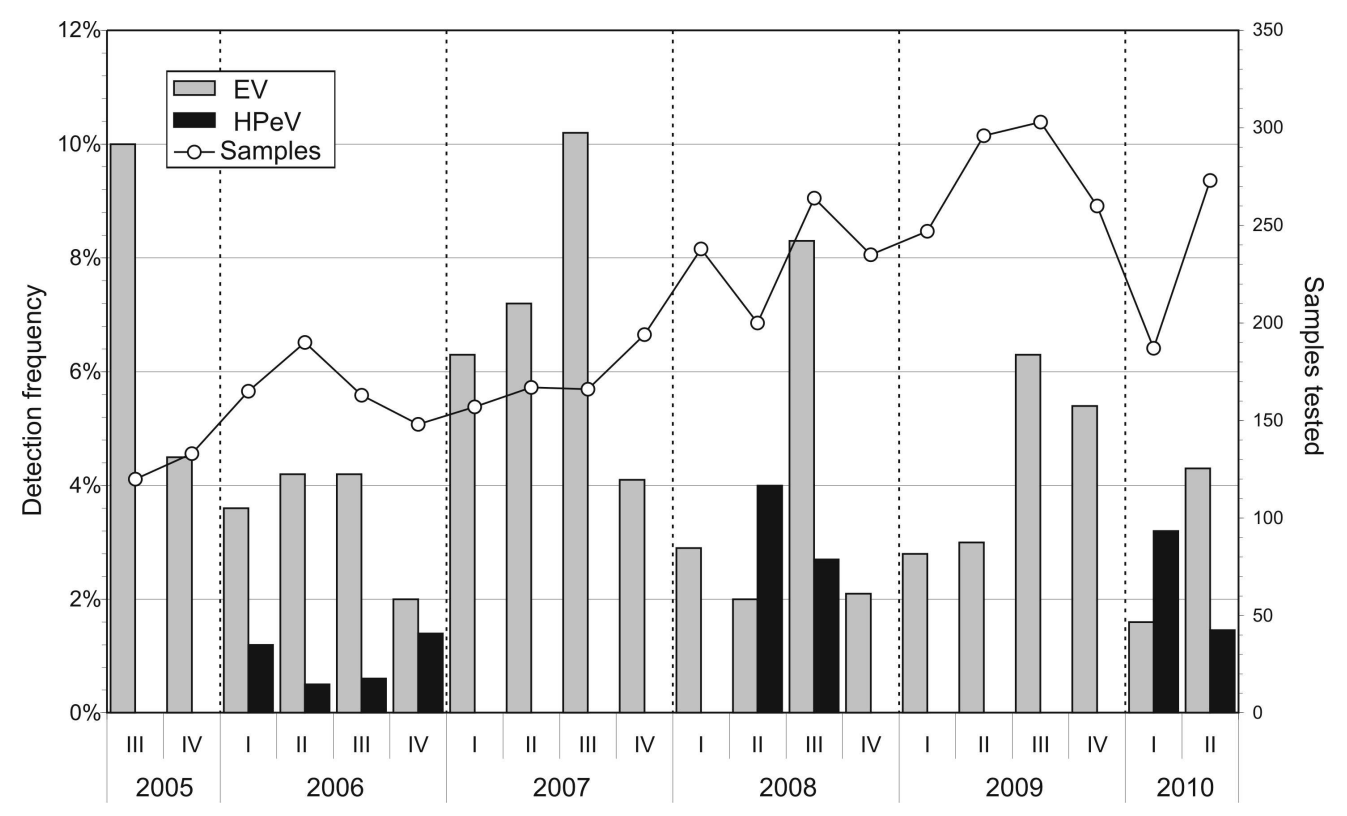

Fig. 1 


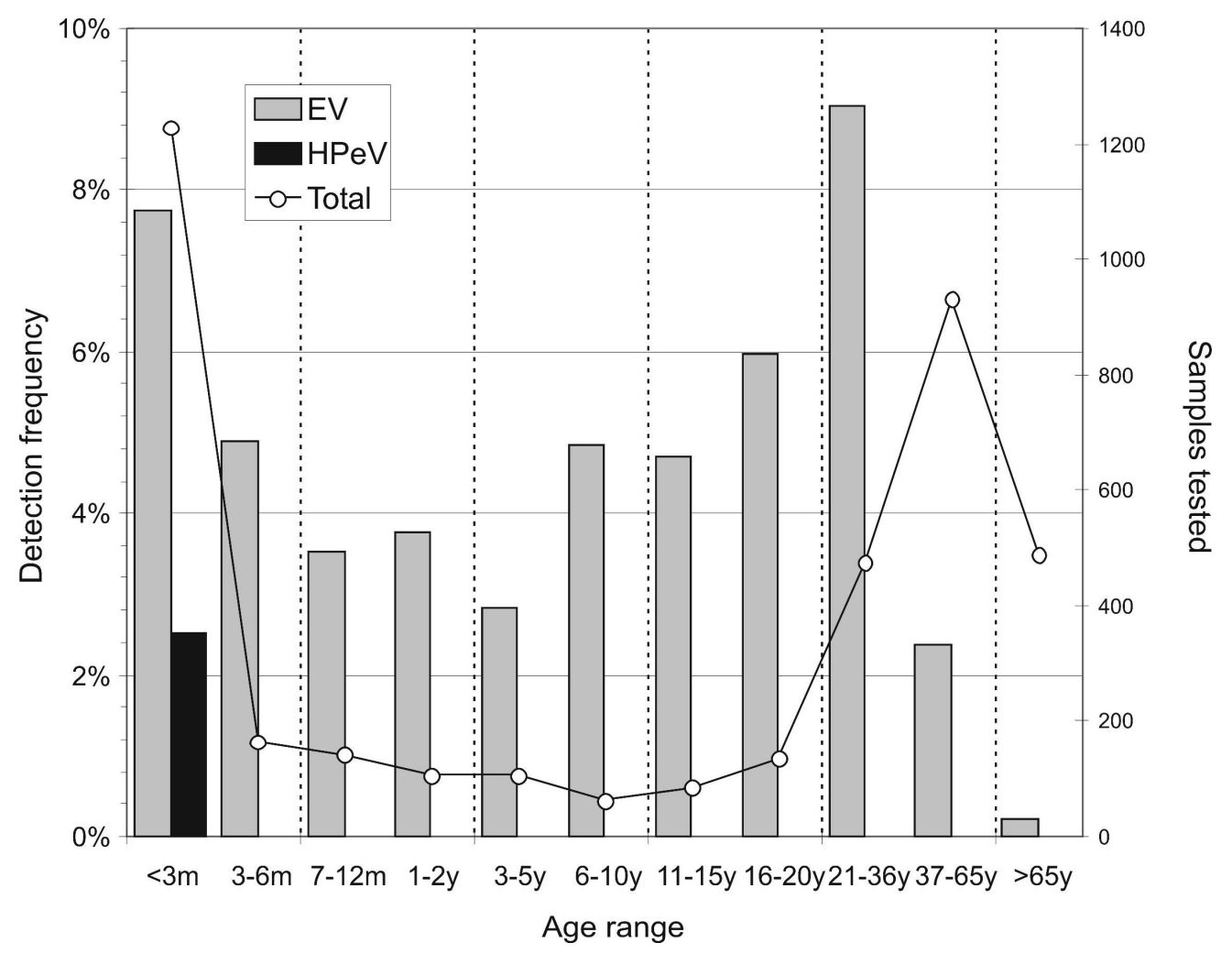

Fig. 2 


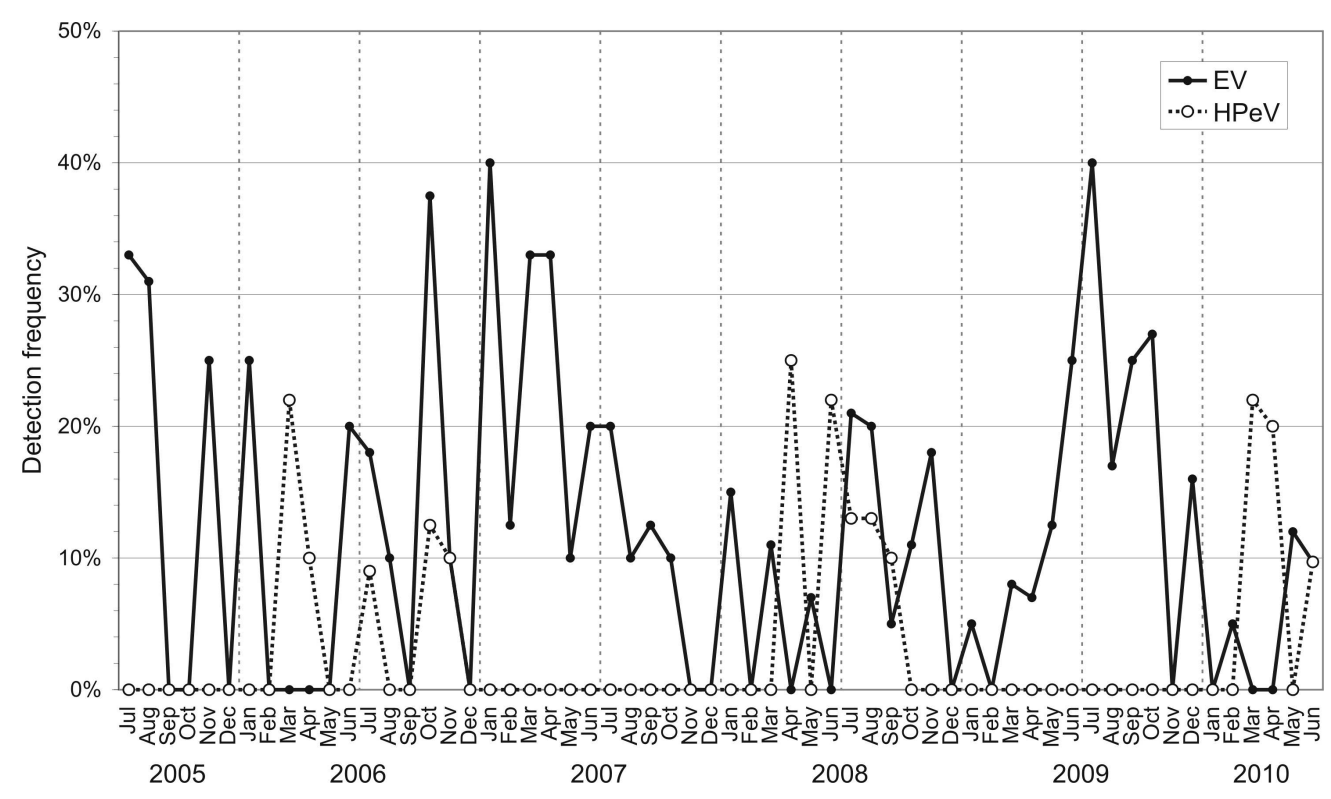

Fig. 3 


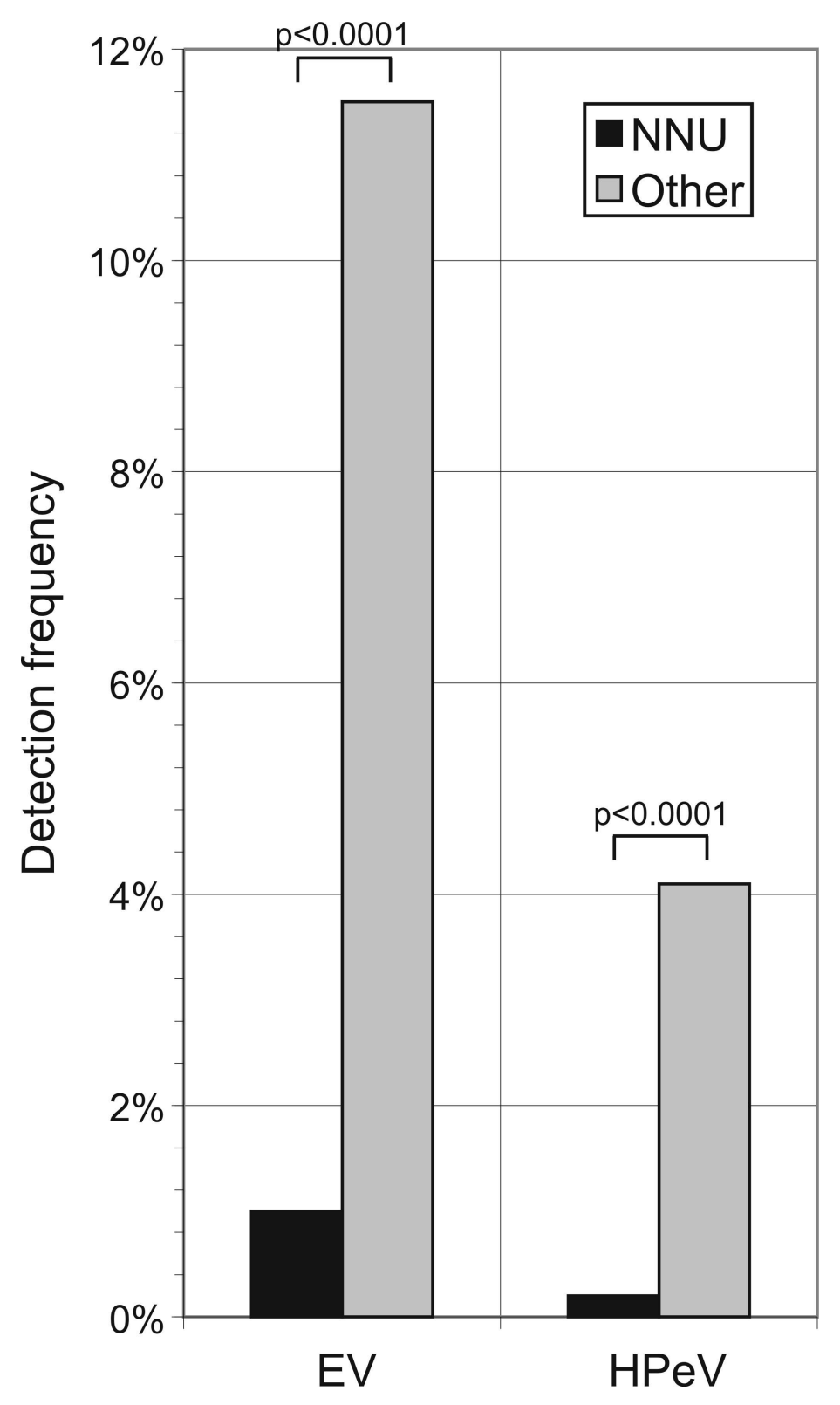

Fig. 4 


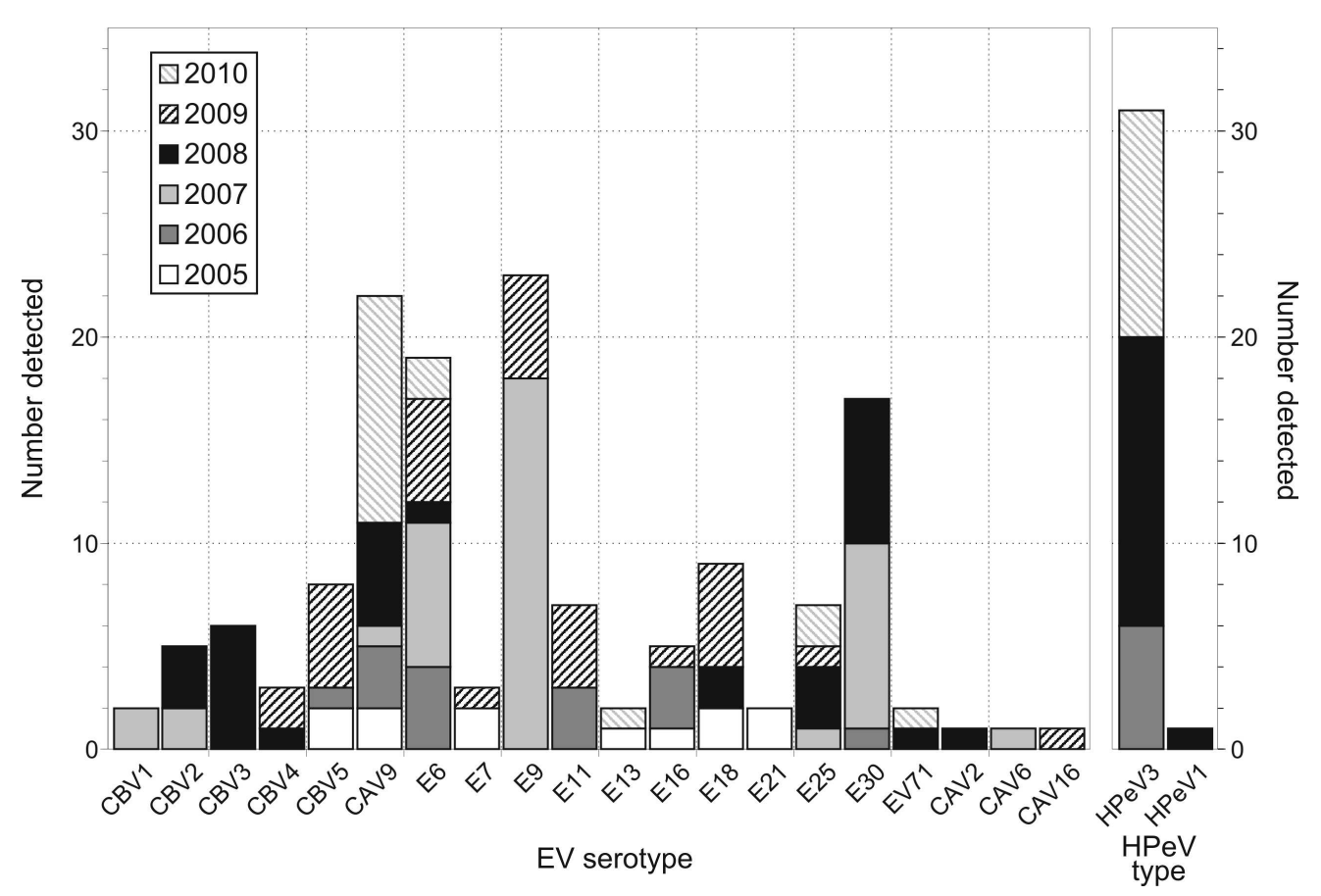

Fig. 5 


\title{
Comparison of human parechovirus and enterovirus detection frequencies in cerebrospinal fluid samples collected over a 5-year period in Edinburgh - HPeV type 3 identified as the most common picornavirus type
}

\author{
Heli Harvala ${ }^{1,2^{*}}$, Nigel McLeish ${ }^{2}$, Jasmina Kondracka $^{2}$, Chloe L. McIntyre ${ }^{2}$, \\ E. Carol McWilliam Leitch ${ }^{2}$, Kate Templeton ${ }^{1}$ and Peter Simmonds ${ }^{2}$ \\ ${ }^{1}$ Specialist Virology Centre, Royal Infirmary of Edinburgh, Edinburgh, EH16 4SB; \\ ${ }^{2}$ Centre for Infectious Diseases, University of Edinburgh, Edinburgh, EH9 1QH, UK
}

Running title: Enterovirus and parechovirus surveillance

*Corresponding Author Dr Heli Harvala

Specialist Virology Centre

Royal Infirmary of Edinburgh

51 Little France Crescent

Edinburgh EH16 4SA, UK

Phone: +44 1312426075

Email: heli.simmonds@hotmail.com

This work was performed at the Centre for Infectious diseases, University of Edinburgh and at the Specialist Virology Centre, Royal Infirmary of Edinburgh, Edinburgh, UK. 


\begin{abstract}
Human enteroviruses (EVs) and more recently parechoviruses (HPeVs) have been identified as the principal viral causes of neonatal sepsis-like disease and meningitis. The relative frequencies of specific $\mathrm{EV}$ and $\mathrm{HPeV}$ types were determined over a 5-year surveillance period using highly sensitive EV and HPeV PCR assays for screening 4168 cerebrospinal fluid (CSF) specimens collected from hospitalised individuals between 2005-2010 in Edinburgh. Positive CSF samples were typed by sequencing of VP1. From the $201 \mathrm{EV}$ and $31 \mathrm{HPeV}$ positive (uncultured) CSF samples on screening, a high proportion of available samples could be directly typed $(176 / 182,97 \%)$. Highest frequencies of EV infections occurred in young adults $(n=43 ; 8.6 \%)$ although a remarkably high proportion of positive samples $(n=98 ; 46 \%)$ were obtained from young infants ( $<3$ months). HPeV infections were seen exclusively in children under the age of three months $(31 / 1105 ; 2.8 \%)$, and confined to spring on even-numbered years (22\% in March 2006, 25\% in April 2008 and 22\% in March 2010). In contrast, EV infections were distributed widely across the years. Twenty different EV serotypes were detected; E9, E6 and CAV9 being found most frequently, whereas all but one $\mathrm{HPeVs}$ were type 3 . Over this period, HPeV3 was identified as the most prevalent picornavirus type in CNS-related infections with similarly high incidences of EV infection frequencies in very young children. The highly sensitive virus typing methods applied in this study will assist further EV and $\mathrm{HPeV}$ screening of sepsis and meningitis cases as well as in future molecular epidemiological studies and population surveillance.
\end{abstract}

\title{
Keywords
}

Sepsis, meningitis, surveillance, neonatal, serotype, molecular diagnostics 


\section{Introduction}

Human enteroviruses (EV) and parechoviruses (HPeV), members of the virus family Picornaviridae, are important human pathogens responsible for a wide spectrum of illnesses varying from the common cold to severe infections of the central nervous system (CNS) and myocardium. Enteroviruses have been divided traditionally into polioviruses (PVs, 3 serotypes), coxsackie A viruses (CAVs, 23 serotypes), coxsackie $\mathrm{B}$ viruses (CBVs, 6 serotypes) and echoviruses (E, 28 serotypes), mainly on the basis of their pathogenicity in laboratory animals. Enteroviruses discovered more recently have not been classified into these subgroups, nor have their antigenic properties been examined in detail; instead they are assigned as enterovirus types numbered chronologically in order of their identification (33 types by the end of 2009). The 93 human EVs fall into 4 genetically distinct species, HEV-A to D (Hyypia et al., 1997, Stanway et al., 2005), with species B variants (including echoviruses, CBVs and CAV9) being the viral cause of CNS-associated infections in Europe identified most frequently.

Along with this reorganisation of enteroviruses was the recognition that two echoviruses, E22 and E23, were different genetically and biologically from other members in the Enterovirus genus. These two serologically distinct viruses were originally isolated and characterised over 50 years ago during a summer diarrhoea outbreak in America (Wigand \& Sabin, 1961). However, they were recognised as genetically distinct from enteroviruses in the early 1990s (Hyypia et al., 1992) and subsequently reassigned to a new picornavirus genus, Parechovirus (King et al., 1999) as human parechovirus (HPeV) types 1 and 2. Since then, a further twelve types (HPeV3 to 14) known to infect human have been discovered (Harvala et al., 2010). 
Although $\mathrm{HPeV}$ infections are common and the clinical course is usually mild, they have been recently associated with sepsis-like disease in very young children (Boivin et al., 2005, Harvala et al., 2009, Wolthers et al., 2008).

There is a high incidence of EV and $\mathrm{HPeV}$ infections in early childhood, as indicated by the high seroprevalence by the time children reach school age (JokiKorpela \& Hyypia, 2001, Tauriainen et al., 2007). Although these infections are mostly asymptomatic, newborn infants are considered to be at a higher risk of severe $\mathrm{EV}$ (and $\mathrm{HPeV}$ ) infection because of the immaturity of their immune system. Previous reports suggest that the risk of symptomatic neonatal EV and $\mathrm{HPeV}$ disease varies by infecting type, and that $\mathrm{CBVs}$, E11 and $\mathrm{HPeV} 3$ are most commonly associated with severe neonatal sepsis-like illness (Harvala et al., 2009, Khetsuriani et al., 2006a, Khetsuriani et al., 2006b). However, fever in young infants can be bacterial in origin and thus it often necessitates a complete set of investigations including blood and urine culture, lumbar puncture as well as the initiation of broad-spectrum antibiotic therapy. This may be avoided in many cases if a rapid diagnosis of EV and HPeV infections could be made.

Traditionally, the diagnosis of EV or HPeV infections had relied on obtaining a positive viral culture from stool, nasopharyngeal swab or cerebrospinal fluid (CSF) samples. This often slow, laborious and insensitive virus detection method has been largely replaced by nucleic acid amplification methods such as reverse-transcriptase polymerase chain reaction (RT-PCR) for detection of viral RNA (Arola et al., 1996, Chapman et al., 1990, Hyypia et al., 1989, Olive et al., 1990, Zoll et al., 1992). Typically, RT-PCR assays target the 5'untranslated region, highly conserved between all four EV species, and between all known types of $\mathrm{HPeV}$. Although extremely useful for virus screening, amplicon sequences from the 5'NCR provide little or no 
information on the (sero)type of the infecting virus. Reliable type identification therefore depends on amplification and genetic analysis of structural gene regions such as VP1. Because of sequence variability in these coding regions, implementation of reliable typing assays for both $\mathrm{EVs}$ and $\mathrm{HPeV}$ requires considerably greater optimization of primer design and amplification conditions to achieve the sensitivity required for direct use on clinical specimens, particularly CSF. However, it is recognized that molecular typing is important as a necessary part of ongoing poliovirus surveillance but also for the molecular epidemiological purposes and for better understanding of symptoms and clinical outcomes. This study describes the large-scale implementation of previously developed highly sensitive typing assays for EVs and $\mathrm{HPeV}$ to investigate the (sero)types and epidemiology of these viruses over the past five years in a large Scottish Virology Centre. 


\section{Methods}

Clinical Samples. A total of 4168 CSF samples referred to the Specialist Virology Centre in Edinburgh during the five-year period (July 2005 - June 2010) were included in this study. Most of the samples were obtained from patients ranging from newborn babies ( $<3$ months) to elderly ( $>65$ years of age) admitted to one of the main University Hospitals (Royal Infirmary Edinburgh [RIE], Western General Hospital [WGH] or Royal Hospital for Sick Children [RHSC]) or to local district hospitals in the Edinburgh area. CSF samples were referred primarily for screening from patients with suspected meningitis or neonatal sepsis-like disease. All CSF samples were screened for herpes simplex virus type 1 (HSV1) and 2 (HSV2) and varicella zoster virus (VZV) by multiplex nested-PCR or real-time PCR, and for enteroviruses by separate RT-PCR (Welch et al., 2001). Real-time RT-PCR was introduced into routine use in the beginning of 2009 (modified from (Dierssen et al., 2008), at the same time screening of CSF samples (total of 2000) for mumps virus by real-time RTPCR was initiated (Krause et al., 2006).

For $\mathrm{HPeV}$ screening and type identification, extracted RNA from 3739 anonymised CSF samples (representing over $90 \%$ of all CSF sample obtained during the period) were deposited in the Specialist Virology Centre CSF sample archive and tested for HPeVs by RT-PCR (Harvala et al., 2008, McLeish et al. manuscript in preparation). Samples were anonymised and archived according to protocol approved by the Lothian Regional Ethics Committee (08/S11/02/2). Stored data included age band, recorded clinical information, referral source, month of sample collection and results of routine virological testing of the sample. 
Amplification of VP1 sequences. Sufficient volumes of CSF were available for typing from 159 of the $201 \mathrm{EV}$-positive samples. Extracted RNA was amplified by a combined RT and first round PCR using the Superscript III (Invitrogen) followed by a second amplification reaction with nested primers specific for species A and B VP1 sequences as previously described (Leitch et al., 2009). Positive HPeV samples were amplified in the VP3/VP1 junction region and sequenced between nucleotide positions 2182 and 2437 to identify $\mathrm{HPeV}$ types as described previously (Harvala et al., 2008).

Nucleotide sequencing and serotype identification. Amplified DNA was directly sequenced using the BigDye Terminator kit (Applied Biosystems, Warrington, UK). Sequences were edited and aligned using the Simmonics sequence editor (http://www.virus-evolution.org). Sequences have been assigned GenBank accession numbers xxxxx-yyyyy. 


\section{Results}

Study group. A total of 4168 CSF samples obtained from 3886 different individuals (1974 males and 1893 females, 19 unknown) were referred predominantly from very young children (age < 3 months; $\mathrm{n}=1248$ [30\%]), middle-aged adults (age 37-65 years; $n=915[22 \%]$ ) and older patients (age > 65 years; $n=499$ [12\%]) during the 5year study period. Routine screening detected HSV1 in 21 samples, HSV2 in 12 samples, VZV in 21 samples, mumps virus in 2 samples and EV in 201 samples resulting overall $6 \%$ frequency of routine virus detection.

Frequency of HPeV and EV infections. Screening of the 3739 archived CSF samples detected HPeV RNA in a total of 31 individual specimens obtained from 31 different study subjects; $6(1.0 \%)$ positive samples were obtained in 2006, 14 (1.7\%) in 2008 and $11(2.2 \%)$ in 2010 (Fig. 1). However, there were marked annual changes in the incidence of HPeV over the study period, with HPeV entirely absent on CSF screening in odd years (a total of 1828 CSF samples screened in 2005, 2007 and 2009; Fig. 1). In comparison, EV was detected throughout the 5-year study period in a total of 201 specimens: $18(7.1 \%)$ in 2005, $27(4.1 \%)$ in 2006, 47 in 2007, $39(4.2 \%)$ in 2008, $50(4.5 \%)$ in 2009 and $20(3.8 \%)$ in 2010.

Epidemiological and clinical associations of $\mathrm{HPeV}$ and $\mathrm{EV}$ infections. All children infected with $\mathrm{HPeV}$ were young infants $(<3$ months of age, Fig. 2) with an average detection frequency in this subject group of $2.8 \%$ (31 of 1105). In contrast, EV infections were widely distributed among most age groups, with the highest frequency seen in young adults aged between 21 and 36 years $(43 / 510 ; 8.6 \%)$. However, a 
remarkably high percentage of all EV infections $(94 / 201 ; 47 \%)$ were found in children under the age of three months (Fig. 2). The highest frequency of HSV1 and VZV infections was found in study subjects over 65 years of age, whereas HSV2 and Mumps virus infections occurred predominantly in the younger adults group (21-36 years and 37-65 years; data not shown). There was no significant over-representation of males among those infected with EV (103 male, 97 female) or HPeV (16 male, 15 female).

Adults infected with EV presented almost exclusively with suspected viral meningitis, whereas young children with $\mathrm{HPeV}$ and $\mathrm{EV}$ infections were referred with fever and sepsis-like illness (Table S1; Supplementary Material). Overall, HPeV presented with greater disease severity; four of $31(13 \%) \mathrm{HPeV}$-positive subjects were treated on the High-Dependency / Intensive Care Units at time sampling compared to 5 from 198 (2.5\%) infected with EV ( $p=0.023$; Fisher's Exact Test).

$\mathrm{EV}$ and HPeV infections in young infants. The remarkable high incidence of $\mathrm{HPeV}$ infections in children under the age of three months were largely confined to spring time on even years (22\% in March 2006, 25\% in April 2008 and 22\% in March 2010; Fig. 3), whereas EV infections were absent in this age group during these months. The prevalence of EV infections (11.8\%) in young infants ( $<3$ months) generally exceeded that of $\mathrm{HPeV}$ infections (4.1\%). Interestingly, the frequency of EV infections was $12.0 \%(88 / 748)$ in children under the age of 3 months referred from home, while only $6 \mathrm{EV}$ infections were diagnosed in $586(1 \%)$ neonates remaining in hospital after birth in RIE Neonatal Unit (NNU) ( $p<0.001$; Fig. 4). A similar discordance in HPeV incidence was observed between these two groups with a $4 \%$ detection frequency 
(30/748) in the former group compared to $0.2 \%(1 / 485 ; p<0.001$; Fisher's Exact Test) among neonates remaining in the NNU).

EV and HPeV type identification. To identify infecting EV serotypes, amplification of VP1 gene was performed. Regions amplified in VP1 allowed previously assigned EV serotypes to be reliably identified (Leitch et al., 2009). An extremely high proportion of (uncultured) CSF samples could be directly typed for EVs (145/151; 96\%), despite the availability of very limited CSF sample volumes and frequently low viral loads. Six samples failed to be detected by VP1 primers, but their identity as species A/B serotypes was confirmed by sequencing the 5'UTR. For $\mathrm{HPeV}$, all 5'UTR screening positive CSF samples (31/31) could be amplified and typed in the VP3/VP1 region.

A total of 16 different species B and 4 species A EV serotypes were detected in this study. The most frequently detected enterovirus types were E9 (23; 15\%), CAV9 $(22 ; 14 \%)$, E6 $(19 ; 13 \%)$, E30 $(17 ; 11 \%)$, E18 $(9 ; 6 \%)$ and CBV5 $(8 ; 5 \%)$ serotypes, along with occasional detections of species A serotypes CAV2, CAV6, CAV16 and EV71 (Fig. 5). Rapid changes in serotype frequencies were observed. Almost all EVs were typed as E9 in early 2007, and were replaced entirely by E30 and E6 in the latter half of the year. No virus predominated in 2005, 2006, 2008 and 2009, where 7, 6, 9 and 11 different serotypes circulated respectively. Most recently in 2010, CAV9 became the predominant serotype. All except one of the HPeV types identified were HPeV3 (30/31), whereas the remaining one was HPeV type 1B. Over the 5-year study period, HPeV3 was the most prevalent picornavirus in CNS-related infections. 
Each serotype infected a wide age range, although E6 and E30 infections were largely or entirely absent in children less than three months of age (data not shown). Similarly to HPeV3 infections (30/30), all E16 infections (5 patients) as well as most E25 $(6 / 7 ; 86 \%)$ and E11 $(5 / 7 ; 71 \%)$ infections were seen in young infants $(<3$ months of age). Most very young children infected with EV or HPeV presented with sepsislike disease, whereas older children and adults commonly had meningitis (Table S1; Supplementary data). All EV infections were reported to the Health Protection Agency, England. Furthermore, HSV2, VZV and mumps virus were associated with meningitis and HSV1 with encephalitis (data not shown). 


\section{Discussion}

This study reports the results of comprehensive screening of CSF samples for enteroviruses and parechoviruses over a 5-year period using molecular techniques for detection and virus characterization. Because of the equivalent sensitivity of the screening PCR for HEV-A and HEV-B viruses (as well as for HEV-C and HEV-D), our rates of detection are uninfluenced by variability in ease of in vitro culture, an important drawback of previous enterovirus surveillance reports that relied on prior virus isolation for identification of serotypes. Similarly, PCR offered a truer reflection of the relative importance of parechoviruses in CNS-related disease; HPeVs, particularly type 3 , are known to be refractory frequently to in vitro culturing (Benschop et al., 2010).

Enterovirus diversity. Over the 5-year study period, the most common enterovirus serotypes identified in Edinburgh were E9, E6, CAV9, E30 and E18. Three of these (E9, E30 and CAV9) were also among the most abundant serotypes isolated from clinical specimens in the UK previously in 1975 to 1994 (Maguire et al., 1999). E6, E9 and E30 were also among the most frequently reported serotypes in the United States in 1970 to 2005 (Khetsuriani et al., 2006a), and similarly E30 and E6 in continental Europe in 2000s (Antona et al., 2007, Blomqvist et al., 2008). In contrast to most previous surveillance data based on virus isolation outside South-East Asia, a total of four different species A enterovirus serotypes were detected (CAV2, CAV6, CAV16 and EV71) in association with neonatal sepsis. This higher frequency of species A variants is consistent with a previous study of viraemia frequency in Scottish blood donors, where CAV16 and EV71 were amongst the identified EV types 
identified most frequently despite never being isolated contemporaneously by the virus diagnostic services serving the same catchment area (Welch et al., 2001). Other evidence for the widespread circulation of EV species A variants is provided by the frequent molecular detection of EV71, CAV6, CAV10 and CAV4 in faecal sample screening of healthy Norwegian infants (Witso et al., 2006) and in Finnish national surveillance (Blomqvist et al., 2008).

Clearly, the adoption of PCR-based methods for virus detection provides a better opportunity to assess population enterovirus diversity. Despite this, no individuals were found to be infected with species C or D EVs, an observation that suggests either their much more restricted community incidence or a greatly reduced likelihood of infected individuals developing the severe disease manifestations that would lead to diagnostic virology testing. The recently implemented weekly screening of sewage in the Edinburgh area has indeed revealed the presence of numerous species $\mathrm{C}$ serotypes (Calvert et al., unpublished observation) providing evidence in favour of the latter explanation (despite the evident neurotropism of the now eradicated polioviruses).

Detection of HPeV3. In contrast to the diversity of enteroviruses detected in CSF, all but one of the 31 parechovirus-positive samples identified were type 3 . Over the 5year study period, it was the most prevalent picornavirus type in CNS-related infection. Comparison of genetic variants in the current study with those detected in previous surveillance provides evidence for a major change in parechovirus molecular epidemiology; HPeV1 (named as E22 at that time and thus part of enterovirus surveillance) was the second most prevalent serotype in the UK detected by virus isolation between 1975 and 1994 (Maguire et al., 1999). Similarly, HPeV1 was 
detected frequently in other parts of Europe and in the United States in the 1980's (Hovi et al., 1996, Khetsuriani et al., 2006a). Although it could be argued that the lack of $\mathrm{HPeV} 3$ detection in these earlier surveys directly resulted from putative insensitivity of virus isolation for this virus type (Benschop et al., 2010), it is nevertheless clear that a major change in either the community circulation of $\mathrm{HPeV} 1$ or its disease outcomes has occurred over the last 3 decades.

HPeV3 infections differed considerably in their seasonality from enteroviruses, being detected exclusively in CSF samples collected in Edinburgh in 2006, 2008 and 2010 (Fig. 3). This biennial cycle is consistent with previous reports of much higher frequencies of $\mathrm{HPeV} 3$ infections in even-numbered years between 2000 and 2006, and the virtual absence in intervening years (Benschop et al., 2008, van der Sanden et al., 2008). To an extent, this periodicity resembles the regular increases in reported cases of several of the commonly circulating EV serotypes. For example, E30 shows a 4-5 year periodicity based on both USA and European surveillance data (Antona et al., 2007, Khetsuriani et al., 2006a, Trallero et al., 2010), while E9 shows a 2-3 year cycle. The re-appearance of E30 and E9 variants after an eclipse phase is associated with the emergence of novel recombinant genetic lineages of the viruses (McWilliam Leitch et al., 2009, McWilliam Leitch et al., 2010). This is however not the case in $\mathrm{HPeV} 3$; the variants circulating in 2006 and 2008 were almost identical in the VP3/VP1 region and sequence analysis of the 3Dpol region did not provide any evidence of the emergence of novel recombinant forms over the ellipse period (Calvert et al., 2010). The phenomena underlying the biennial cycle of $\mathrm{HPeV} 3$ infections and its much greater frequency in spring thus remains to be determined. 
CNS-associated infections in young children. There were several differences in the age distribution between different EV serotypes and HPeVs. A remarkably high proportion of all EV infections (47\%) were observed in infants younger than three months of age. This is higher than reported previously; enterovirus infection in the neonatal period accounted for about $10 \%$ of the total number of reported cases of enterovirus infections in the United States, whereas children $<1$ year of age accounted for $44 \%$ of reports (Khetsuriani et al., 2006a). National surveillance data from France and the UK has shown that the vast majority of EV infections occur in young children, with infants below the age of one year accounting for about a third of all cases (Antona et al., 2007, Maguire et al., 1999).

As found in the current study, it has also been shown that the relative frequency and predominant serotypes of enteroviruses affecting neonates differ from the pattern observed in the general population. Indeed, the remarkable variability in the proportion of young infants infected with enteroviruses could be partly accounted by seasonal changes in the circulation of serotypes associated with CNS-related disease in young infants. These include CBVs, E11, E16 and E25, each of which typically target neonates (Verboon-Maciolek et al., 2003), and E9 and E30 which infect neonates much less frequently than older persons (Khetsuriani et al., 2006a, Khetsuriani et al., 2006b). In this study, the lowest proportions of EV infections in young infants ( $<3$ months of age; $36 \%$ ) were observed in 2007, the year when E9 and E30 were the two viruses detected most frequently, accounting for $57 \%$ of all EV infections. Conversely, the highest proportion of young infants $(<3$ months of age) infected with EV (57\%) occurred in 2009, when CBVs, and E11 were amongst the two most prevalent viruses. Furthermore, E16 was positively associated with infections in very young children ( $<3$ months; all infections in this age group). 
Although marked differences in the age distribution of different EV serotypes were observed, an even more polarised age distribution was observed in $\mathrm{HPeV}$ infections. These were detected exclusively in CSF samples obtained from young infants ( $<3$ months), and entirely absent from older children and adults. These findings are consistent with the previous analysis of $\mathrm{HPeV}$ infection (Harvala et al., 2009); the parallel observation that $\mathrm{HPeV} 3$ detection in respiratory samples was similarly age-restricted providing evidence that the virus circulation is limited to children in this very young age group. This association is therefore not the result of biased sampling and greater likelihood of sampling because of disease severity, or a clinical need to identify viral from bacterial causes of sepsis.

The observation that both $\mathrm{EV}$ or $\mathrm{HPeV}$ infections were restricted almost entirely to infants who had been admitted to hospital from home (Fig. 4) and were almost entirely absent from children in the same age range who had remained hospitalised after birth, provides clear evidence for community sources of infection that are largely curtailed in a hospital setting. These observations reassure that nosocomial transmission of $\mathrm{EVs}$ and $\mathrm{HPeV}$ in the Edinburgh neonatal unit was extremely uncommon over the five-year study period. The findings also indicate how diagnostic screening for EVs and $\mathrm{HPeV}$ may be targeted more effectively as there would be a case to exclude those who have remained in hospital after birth from testing.

Why has the incidence of neonatal EV and HPeV infections increased? One of the major findings in the current study was the remarkable frequency of EV and HPeV infections in children less than three months old, presenting with sepsis-like illnesses. The observed frequencies are much higher than reported previously in this age range 
for both virus groups (Figueroa et al., 1989, Khetsuriani et al., 2006a, Legay et al., 2002a), and cannot be entirely attributed to improvement in the effectiveness of screening methodologies (although the greater ability of PCR to detect HPeV3 and species A EVs compared to virus culture undoubtedly contributed partly to the increased detection frequency in CSF).

In seeking to explain this change, it might be proposed that $\mathrm{HPeV} 3$ and several of EVs specifically found in neonates may have changed in their biological properties (associated with a greater propensity to infect systemically), in their epidemiology and transmission, or that there have been systematic changes in host susceptibility over the past decades. One factor supporting the latter hypothesis is the evidence for a progressive lowering of maternal seroprevalence and lack of passively transferred protective neutralizing antibodies transferred passively in an increasing proportion of neonate and infants. The changing epidemiology of enterovirus infections has indeed been demonstrated clearly in Finland (Viskari et al., 2000): the proportion of pregnant women lacking antibodies to CBV4 increased from 6\% in 1983 to $17 \%$ in 1995 $(\mathrm{p}<0.001)$. Increasing number of young infants will therefore lack the protective maternal antibodies to viruses circulating in the community, and increase the likelihood of developing severe CNS-associated (sepsis-like) EV disease.

Whether such changes in the viral circulation account for the increasing incidence of $\mathrm{HPeV} 3$-associated sepsis-like illness in neonates is unclear, as are the underlying reasons for the major clinical and epidemiological differences from HPeV1, which is well documented to cause asymptomatic or mild infections and shows a strikingly high incidence in the first two years of life (Joki-Korpela \& Hyypia, 1998, Tapia et al., 2008, Tauriainen et al., 2007). The close to universal adult seropositivity for $\mathrm{HPeV} 1$ contrasts with that of $\mathrm{HPeV} 3$; over $30 \%$ of child-bearing 
aged women has been shown to lack antibodies to HPeV3 (Ito et al., 2004). However, unlike the situation with EVs, the lower adult seroprevalence of $\mathrm{HPeV} 3$ may have resulted directly from its relatively recent emergence compared to other $\mathrm{HPeV}$ types. Through analysis of the genetic diversity of circulating currently and historical isolates of $\mathrm{HPeV} 3$, it has been estimated that a common ancestor for this type appeared in the human population as recently as 1987 (Calvert et al., 2010), approximately 10 years before it was first isolated in Japan and Canada (Abed \& Boivin, 2005, Ito et al., 2004). HPeV3 may therefore have not spread sufficiently far into adult human populations to provide the essential levels of maternal protection required to prevent neonatal disease.

This proposed scenario contrasts with $\mathrm{HPeV1}$, where close to universal maternal seropositivity may account for the current almost complete lack of neonatal disease associated with this type. Sewage surveillance in Edinburgh indeed documents the predominance of $\mathrm{HPeV} 1$ over other $\mathrm{HPeV}$ types circulating in the local community (Calvert et al., unpublished observations). Interestingly, diagnosed HPeV1 infections were substantially more frequent and more severe in the 1980s (Figueroa et al., 1989, Koskiniemi et al., 1989, Legay et al., 2002b), where it accounted for close to $8 \%$ of all $\mathrm{HPeV} / \mathrm{EV}$ infections in national surveillance systems (Antona et al., 2007, Khetsuriani et al., 2006a, Khetsuriani et al., 2006b, Maguire et al., 1999). We might speculate that the emergence of HPeV1 simply preceded that of $\mathrm{HPeV} 3$ and these disease presentations originated similarly from a lack of universal maternal exposure at that time.

Combined, these findings emphasises the importance of ongoing surveillance of EVs and $\mathrm{HPeVs}$ in our understanding of human picornavirus-related disease. In particular, the evidence for relatively recent emergence and marked changes in the 
Harvala et al., (Manuscript)

circulation of different $\mathrm{HPeV}$ and $\mathrm{EV}$ types over often short periods provide the basis for more focused investigations of the role of population immunity, community circulation and prevalence in the population. 


\title{
References
}

\begin{abstract}
Abed Y, Boivin G. 2005. Molecular characterization of a Canadian human parechovirus (HPeV)-3 isolate and its relationship to other HPeVs. J Med Virol $77: 566-570$.
\end{abstract}

Antona D, Leveque N, Chomel JJ, Dubrou S, Levy-Bruhl D, Lina B. 2007. Surveillance of enteroviruses in France, 2000-2004. Eur J Clin Microbiol Infect Dis $26: 403-412$.

Arola A, Santti J, Ruuskanen O, Halonen P, Hyypia T. 1996. Identification of enteroviruses in clinical specimens by competitive PCR followed by genetic typing using sequence analysis. J Clin Microbiol 34:313-318.

Benschop K, Minnaar RP, Koen G, van Eijk HW, Dijkman K, Westerhuis B, Molenkamp R, Wolthers K. 2010. Detection of enterovirus and human parechovirus genotypes from clinical stiool samples; PCR and direct molecular typing, culture characteristics and serotyping. J Clin Microbiol (in press).

Benschop K, Thomas X, Serpenti C, Molenkamp R, Wolthers K. 2008. High prevalence of human parechovirus genotypes in the Amsterdam region and the identification of specific $\mathrm{HPeV}$ variants by direct genotyping of stool samples. J Clin Microbiol 46:3965-3970.

Blomqvist S, Paananen A, Savolainen-Kopra C, Hovi T, Roivainen M. 2008. Eight years of experience with molecular identification of human enteroviruses. J Clin Microbiol 46:2410-2413. 
Boivin G, Abed Y, Boucher FD. 2005. Human parechovirus 3 and neonatal infections. Emerg Infect Dis 11:103-105.

Calvert J, Chieochansin T, Benschop K, McWilliam-Leitch EC, Drexler JF, Grywna

K, da Costa Ribeiro H, Drosten C, Harvala H, Poovorawan Y, Wolthers K, Simmonds P. 2010. The recombination dynamics of human parechoviruses; investigation of typespecific differences in frequency and epidemiological correlates. J Gen Virol 91:12291238.

Chapman NM, Tracy S, Gauntt CJ, Fortmueller U. 1990. Molecular detection and identification of enteroviruses using enzymatic amplification and nucleic acid hybridization. J Clin Microbiol 28:843-850.

Dierssen U, Rehren F, Henke-Gendo C, Harste G, Heim A. 2008. Rapid routine detection of enterovirus RNA in cerebrospinal fluid by a one-step real-time RT-PCR assay. J Clin Virol 42:58-64.

Figueroa JP, Ashley D, King D, Hull B. 1989. An outbreak of acute flaccid paralysis in Jamaica associated with echovirus type 22. J Med Virol 29:315-319.

Harvala H, Robertson I, McWilliam Leitch C, Chieochansin T, Templeton K, Simmonds P. 2009. Aetiological role of human parechovirus type 3 in neonatal sepsis identified by direct typing assay on cerebrospinal fluid. J Infect Dis 199:1753-1760.

Harvala H, Robertson I, McWilliam Leitch EC, Benschop K, Wolthers KC, Templeton K, Simmonds P. 2008. Epidemiology and clinical associations of human parechovirus respiratory infections. J Clin Microbiol 46:3446-3453. 
Harvala H, Wolthers K, Simmonds P. 2010. Parechoviruses in children:

understanding a new infection. Curr Opin Infect Dis 23:224-230.

Hovi T, Stenvik M, Rosenlew M. 1996. Relative abundance of enterovirus serotypes in sewage differs from that in patients: clinical and epidemiological implications. Epidemiol Infect 116:91-97.

Hyypia T, Auvinen P, Maaronen M. 1989. Polymerase chain reaction for human picornaviruses. J Gen Virol 70:3261-3268.

Hyypia T, Horsnell C, Maaronen M, Khan M, Kalkkinen N, Auvinen P, Kinnunen L, Stanway G. 1992. A distinct picornavirus group identified by sequence analysis. Proc Natl Acad Sci U S A 89:8847-8851.

Hyypia T, Hovi T, Knowles NJ, Stanway G. 1997. Classification of enteroviruses based on molecular and biological properties. J Gen Virol 78 ( Pt 1):1-11.

Ito M, Yamashita T, Tsuzuki H, Takeda N, Sakae K. 2004. Isolation and identification of a novel human parechovirus. J Gen Virol 85:391-398.

Joki-Korpela P, Hyypia T. 1998. Diagnosis and epidemiology of echovirus 22 infections. Clin Infect Dis 27:129-136.

Joki-Korpela P, Hyypia T. 2001. Parechoviruses, a novel group of human picornaviruses. Ann Med 33:466-471.

Khetsuriani N, Lamonte-Fowlkes A, Oberst S, Pallansch MA. 2006a. Enterovirus surveillance--United States, 1970-2005. MMWR Surveill Summ 55:1-20. 
Khetsuriani N, Lamonte A, Oberste MS, Pallansch M. 2006b. Neonatal enterovirus infections reported to the national enterovirus surveillance system in the United States, 1983-2003. Pediatr Infect Dis J 25:889-893.

King AMQ, Brown F, Christian P, Hovi T, Hyypia T, Knowles NJ, Lemon SM, Minor PD, Palmenberg AC, Skern T, Stanway G. 1999. Family Picornaviridae.pp 996. In M. H. V. Van Regenmortel, C. M. Fauquet, D. H. L. Bishop, C. H. Calisher, E. B. Carsten, M. K. Estes, S. M. Lemon, J. Maniloff, M. A. Mayo, D. J. McGeoch, C. R. Pringle, and R. B. Wickner (ed.), Virus taxonomy. Seventh Report of the International Committee for the Taxonomy of Viruses. Academic Press, New York, N.Y..

Koskiniemi M, Paetau R, Linnavuori K. 1989. Severe encephalitis associated with disseminated echovirus 22 infection. Scand J Infect Dis 21:463-466.

Krause CH, Eastick K, Ogilvie MM. 2006. Real-time PCR for mumps diagnosis on clinical specimens--comparison with results of conventional methods of virus detection and nested PCR. J Clin Virol 37:184-189.

Legay V, Chomel JJ, Fernandez E, Lina B, Aymard M, Khalfan S. 2002a. Encephalomyelitis due to human parechovirus type 1. J Clin Virol 25:193-195.

Legay V, Chomel JJ, Lina B. 2002b. Specific RT-PCR procedure for the detection of human parechovirus type 1 genome in clinical samples. J Virol Methods 102:157-160.

Leitch EC, Harvala H, Robertson I, Ubillos I, Templeton K, Simmonds P. 2009.

Direct identification of human enterovirus serotypes in cerebrospinal fluid by amplification and sequencing of the VP1 region. J Clin Virol 44:119-124. 
Maguire HC, Atkinson P, Sharland M, Bendig J. 1999. Enterovirus infections in England and Wales: laboratory surveillance data: 1975 to 1994. Commun Dis Public Health 2:122-125.

McWilliam Leitch EC, Bendig J, Cabrerizo M, Cardosa J, Hyypia T, Ivanova OE, Kelly A, Kroes AC, Lukashev A, Macadam A, McMinn P, Roivainen M, Trallero G, Evans DJ, Simmonds P. 2009. Transmission networks and population turnover of echovirus 30. J Virol 83:2109-2118.

McWilliam Leitch EC, Cabrerizo M, Cardosa J, Harvala H, Ivanova OE, Kroes AC, Lukashev A, Muir P, Odoom J, Roivainen M, Susi P, Trallero G, Evans DJ, Simmonds P. 2010. Evolutionary dynamics and temporal/geographical correlates of recombination in the human enteroviruses, echovirus 9, 11 and 30. J Virol 84:92929300.

Olive DM, Al Mufti S, Al Mulla W, Khan MA, Pasca A, Stanway G, Al Nakib W. 1990. Detection and differentiation of picornaviruses in clinical samples following genomic amplification. J Gen Virol 71:2141-2147.

Stanway G, Brown F, Christian P, Hovi T, Hyypia T, King AMQ, Knowles NJ, Lemon SM, Minor PD, Pallansch MA, Palmenberg AC, Skern T. 2005. Family Picornaviridae.757-778.

Tapia G, Cinek O, Witso E, Kulich M, Rasmussen T, Grinde B, Ronningen KS. 2008. Longitudinal observation of parechovirus in stool samples from Norwegian infants. J Med Virol 80:1835-1842. 
Tauriainen S, Martiskainen M, Oikarinen S, Lonnrot M, Viskari H, Ilonen J, Simell

O, Knip M, Hyoty H. 2007. Human parechovirus 1 infections in young children--no association with type 1 diabetes. J Med Virol 79:457-462.

Trallero G, Avellon A, Otero A, de Miguel T, Perez C, Rabella N, Rubio G, Echevarria JE, Cabrerizo M. 2010. Enteroviruses in Spain over the decade 1998-2007: virological and epidemiological studies. J Clin Virol 47:170-176.

van der Sanden S, de Bruin E, Vennema H, Swanink C, Koopmans M, van der Avoort H. 2008. Prevalence of human parechovirus in the Netherlands in 2000 to 2007. J Clin Microbiol 46:2884-2889.

Verboon-Maciolek MA, Nijhuis M, Van Loon AM, van Maarssenveen N, van Wieringen H, Pekelharing-Berghuis MA, Krediet TG, Gerards LJ, Fleer A, Diepersloot RJ, Thijsen SF. 2003. Diagnosis of enterovirus infection in the first 2 months of life by real-time polymerase chain reaction. Clin Infect Dis 37:1-6.

Viskari HR, Koskela P, Lonnrot M, Luonuansuu S, Reunanen A, Baer M, Hyoty H. 2000. Can enterovirus infections explain the increasing incidence of type 1 diabetes? Diabetes Care 23:414-416.

Welch JB, McGowan K, Searle B, Gillon J, Jarvis LM, Simmonds P. 2001. Detection of enterovirus viraemia in blood donors. Vox Sang 80:211-5.

Wigand R, Sabin AB. 1961. Properties of ECHO types 22, 23 and 24 viruses. Arch Gesamte Virusforsch 11:224-47.:224-247. 
Witso E, Palacios G, Cinek O, Stene LC, Grinde B, Janowitz D, Lipkin WI, Ronningen KS. 2006. High prevalence of human enterovirus a infections in natural circulation of human enteroviruses. J Clin Microbiol 44:4095-4100.

Wolthers KC, Benschop KS, Schinkel J, Molenkamp R, Bergevoet RM, Spijkerman IJ, Kraakman HC, Pajkrt D. 2008. Human parechoviruses as an important viral cause of sepsislike illness and meningitis in young children. Clin Infect Dis 47:358-363.

Zoll GJ, Melchers WJ, Kopecka H, Jambroes G, van der Poel HJ, Galama JM. 1992. General primer-mediated polymerase chain reaction for detection of enteroviruses: application for diagnostic routine and persistent infections. J Clin Microbiol 30:160165. 


\section{Figure legends}

Fig. 1. Referral months of enterovirus (EV) and human parechovirus (HPeV) positive CSF samples showing detection frequencies and total number of tested samples. I=January, February, March; II=April, May, June; III=July, August, September; $\mathrm{IV}=$ October, November, December.

Fig. 2. The positivity rate of $\mathrm{HEV}$ and $\mathrm{HPeV}$ infections in each age category

Fig. 3. The monthly frequency of EV and $\mathrm{HPeV}$ infections in infants under the age of three months during the 5-year surveillance.

Fig. 4. The frequency of EV infection was $11.8 \%$ (87/748) in children under the age of 3 months excluding neonates treated at the NNU, where $6 \mathrm{EV}$ infections was diagnosed (6/586; 1\%) during the 5-year surveillance (July 2005 - June 2010). Similarly, the frequency of HPeV infections varied from $4.1 \%$ (31/748) to $0.2 \%$ (1/586). Both differences were statistically significant ( $\mathrm{p}<0.0001$, Chi-squared test). A total number of CSF sample tested in this age group ( $<3$ months) was 1334 .

Fig. 5. The number of individual HEV serotypes and HPeV types detected over the 5 year period of the study. 